\title{
Reconstruction of open solar magnetic field in 19 th and 20th centuries
}

\author{
V.G. Ivanov and E.V. Miletsky \\ Central Astronomical Observatory at Pulkovo, Saint-Petersburg, Russia; E-mail: \\ solar1@gao.spb.ru
}

Abstract. The reconstructions of the open solar magnetic field since 1844 (with use of geomagnetic data) and since 1915 (with use of $\mathrm{H} \alpha$-spectroheliograms) are obtained and compared $\dagger$.

The large-scale solar magnetic fields modulate parameters of interplanetary medium and play important role in mechanisms of solar-terrestrial links. Unfortunately, systematical direct observations of the large-scale solar magnetic fields began as late as in the second half of the 20th century, and it is of large interest to get information about its behaviour in the earlier epoches.

The coefficients of multipole expansion of the solar photospheric magnetic field by spherical functions $Y_{l m}$, for $l=0 \ldots 9, m=-l \ldots l$ (see Hoeksema \& Scherrer 1986) for time range 1976-2003 are available on site of Wilcox Solar Observatory at Stanford (http://quake.stanford.edu/ wso/Harmonic.los). Using these data, one can calculate the radial component of the magnetic field $B_{r}$ on the source surface of radius $R_{S}=2.5 R_{\odot}$ and to obtain the average open magnetic field (AOMF)

$$
B_{S}=\frac{1}{4 \pi R_{S}^{2}} \int_{S}\left|B_{r}\right| d S .
$$

It can be shown that $B_{S}$ is really determined by the lowest field harmonics (Lean et al. $2002)$, and one can evaluate it with use of only two indices: the axial dipole strength $B_{A D S}(l=1, m=0)$ and the equatorial dipole strength $B_{E D S}(l=1, m= \pm 1)$. The dependence of $B_{S}$ upon the dipoles strengths can be approximated by a linear relation

$$
B_{S} \approx B_{S}^{(D S)}=0.224 B_{A D S}+0.359 B_{E D S},
$$

with correlation coefficient $r\left(B_{S}, B_{S}^{(D S)}\right)=0.96$.

Makarov et al. (2001) reconstructed a longer dataset of the multipole coefficients with use of $\mathrm{H} \alpha$-spectroheliograms for time range 1915-1989. However, the "raw" dipoles strengths $\widetilde{B}^{(H \alpha)}$ got from these reconstruction are to be corrected. Minimizing the error between the "corrected" dipole strengths $B^{(H \alpha)}$ and ones obtained from direct observations $B$ in 1976-1989, one can get for the former evaluative relations

$$
\begin{gathered}
B_{A D S}^{(H \alpha)}=0.696 \widetilde{B}_{A D S}^{(H \alpha)}, \\
B_{E D S} \approx B_{E D S}^{(H \alpha)}=5.4 \cdot 10^{-3} \widetilde{B}_{E D S}^{(H \alpha)} \cdot S_{s p},
\end{gathered}
$$

where $S_{s p}$ are annual total sunspot areas in millionths of solar disk. Therefore, we can calculate AOMF for the time range 1915-1989 with use of relation

$$
B_{S}^{(H \alpha)}=0.224 B_{A D S}^{(H \alpha)}+0.359 B_{E D S}^{(H \alpha)}
$$

(cp. 0.2). The resulting data are presented in Figure (the bold curve).

$\dagger$ The full version of this paper can be found at http://www.gao.spb.ru/personal/ivanov/ papers/iv-mil-iaus2004.pdf 


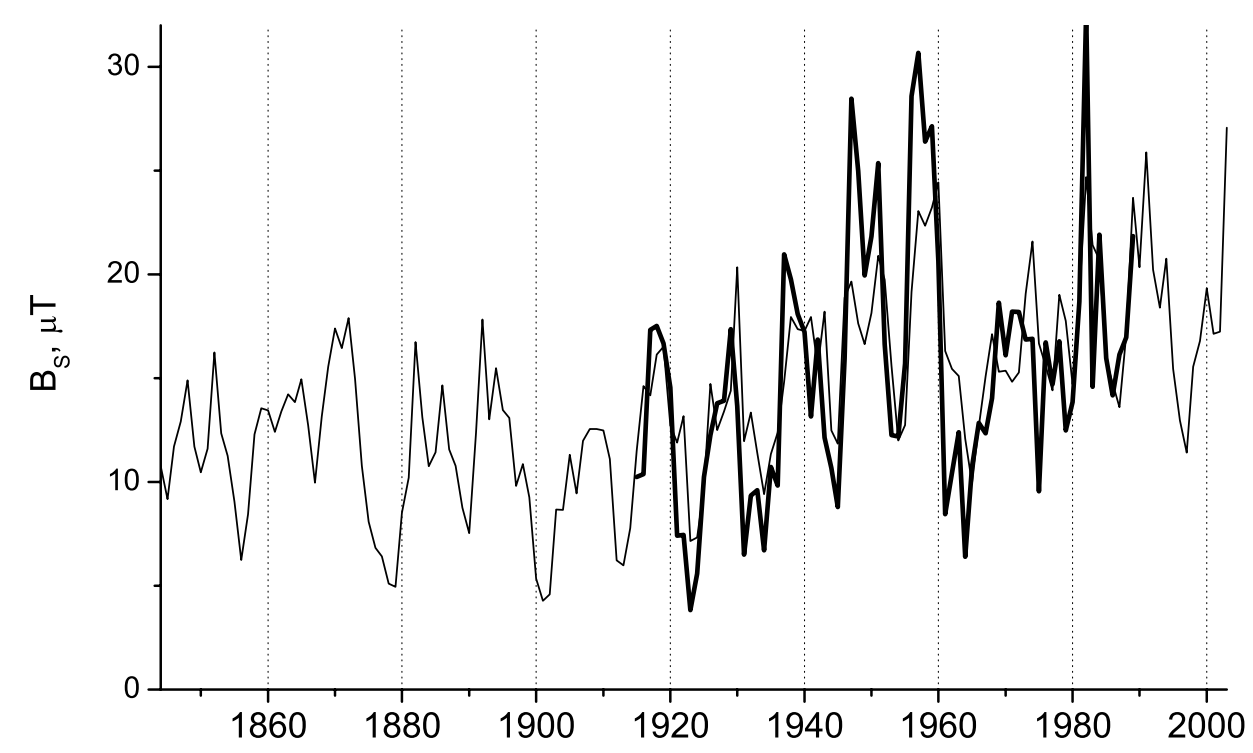

To check the validity of this AOMF reconstruction we can use an alternative approach, exploiting a relation between AOMF and geomagnetic index $a a$ (see Lockwood et al. 1999). The following model

$$
B_{S}^{(a a W)}=0.71 a a+0.014 W
$$

yields correlation with the observed AOMF $r\left(B_{S}^{(a a W)}, B_{S}\right)=0.76$ for the period 19762003. This model, based upon an extended data set of $a a$ index (Nevanlinna \& Kataja 1993, http://www.geo.fmi.fi/MAGN/K-index/), provides us with estimation of AOMF since 1844 (the thin curve in Figure).

The two obtained series prove to be rather similar, especially in their trend component, with correlation $r\left(B_{S}^{(H \alpha)}, B_{S}^{(a a W)}\right)=0.77$ for $1915-1989$. It is important to underline that these two reconstructions are based upon independent information. To build the first model we exploit information about large scale solar magnetic field, while the second one is based upon data of sunspot and geomagnetic activity. We can see that both models exhibit increase of AOMF approximately by a factor of two in the first half of 20th century, confirming thereby the known results (see, e.g., Lockwood et al. 1999).

The work was supported by grants INTAS 2000-0752, INTAS 2001-0550, RFBR 0402-17560 and RFBR 03-02-17505, Federal Program "Astronomy-1105", Program of Presidium of Russian Academy of Sciences "Non-stationary phenomena in astronomy".

\section{References}

Hoeksema, T., \& Scherrer, P.H. 1986 Report UAG-94.

Lean, J.L., Wang, Y.-M., \& Sheeley, N.R. 2002 Geophys. Res. Lett. 29, 2224.

Lockwood, M., Stamper, R., \& Wild, M.N. 1999 Nature 399, 437.

Makarov, V.I., Tlatov, A.G., Callebaut, D.K., Obridko, V.N. \& Shelting, B.D. 2001 Solar Physics 198, 409.

Nevanlinna, H., \& Kataja, E., 1993 Geophys. Res. Lett. 20, 2703. 